\title{
Research on Informatization of Electromechanical System Condition Monitoring and Fault Warning
}

\author{
Ailian Li \\ Shandong Vocational College of Light Industry, Zibo, Shandong, 255300
}

Keywords: Fault Warning, Condition Monitoring, Electromechanical System

\begin{abstract}
With the development of contemporary industry and science and technology, an overall trend in the development of modern equipment is to develop in the direction of complexity, intelligence and automation. In the operation of in-service equipment, failures lead to vicious accidents. The safety and reliability of equipment operation have an important impact on the national economy and people's livelihood, social stability and national resources and environment. The urgency of ensuring the safe and reliable operation of equipment has become increasingly prominent, and the safety of equipment service and maintenance support issues have become more and more important.
\end{abstract}

\section{Introduction}

Electromechanical systems, especially high-end, large-scale, and key electromechanical equipments in manufacturing, are often in poor working conditions, unstable, high-power, heavy-duty and continuous operation. Vicious accidents caused by early fault development sometimes occur, in order to eliminate their faults. Hidden dangers to avoid security accidents, the modern industry urgently needs to adopt the information technology to ensure the safe operation of mechanical and electrical equipment, based on this technology to reveal the evolution of the operating state of electromechanical equipment, and then to conduct early fault prediction. Traditional post-repair maintenance, regular maintenance and other equipment maintenance methods are not only costly but still difficult to effectively prevent and avoid accidents. It is imperative to adopt more scientific maintenance methods, such as adopting modern predictive maintenance methods with information technology as the core technology.

\section{Development of condition monitoring and fault diagnosis system}

With the development of electromechanical system condition monitoring and diagnostic technology, various monitoring and diagnostic instruments and systems have emerged in an endless stream, and their functions are becoming more and more powerful. The vibration measuring instruments and systems are taken as examples. The functions include: shaft vibration monitoring, such as shaft Heart trajectory analysis, axial series motion, peak-to-peak calculation of shaft vibration displacement; shell vibration monitoring; spectrum analysis, such as frequency refinement, order spectrum analysis, order tracking spectrum analysis, 3D power spectrum analysis; automatic pre-alarm, alarm; Fault feature extraction and diagnosis.

This type of system is also called master-slave monitoring and diagnosis system. Sensors are installed at multiple measuring points on the equipment. The data is collected and processed by the on-site microprocessor for each measuring point of the equipment. The status is performed by the professional on the host system. Analysis and fault judgment. Compared with the offline regular monitoring system, although the trouble of changing the measuring point is eliminated, and the detection and alarm can be performed online, the data analysis and judgment need to be performed offline, and the analysis and judgment require the participation of professional technicians. The class system is also called the master-slave monitoring and diagnosis system. The sensors are installed at multiple measuring points on the equipment, and the data is collected and processed by the on-site microprocessor for each measuring point of the equipment. The state analysis is 
performed by the professional on the host system. And fault judgment. Compared with the offline regular monitoring system, although the trouble of changing the measuring point is eliminated, and the detection and alarm can be performed online, the data analysis and judgment need to be performed offline, and the analysis and judgment require the participation of professional technicians.

\section{Research status and key issues of information technology for fault monitoring and early warning of electromechanical systems}

Research and application of domestic and international electromechanical system service monitoring and early warning information technology, mainly for high-end, large-scale and key electromechanical equipment, focusing on solving difficult problems of fault prediction in complex operating conditions such as non-stationary and nonlinear non-stationary and long-term electromechanical equipment These difficult technical problems involve fault signal analysis methods and fault trend prediction methods, including:

1) On the basis of state monitoring and fault diagnosis research, in recent years, fault signal analysis focuses on the cause, type, location and extent of fault occurrence; on the other hand, in order to effectively prevent accidents in advance, low fault SNR in early faults The difficult problems of weak signal analysis have been further concerned and researched, such as: revealing the evolution of the operating state and extracting fault trend information.

2) The traditional fault trend prediction method and predictive maintenance are based on the change of the characteristic quantity of energy (such as the vibration root speed rms value vibration intensity, vibration signal power spectrum, etc.), but many equipments are often subjected to working conditions and loads. The influence of non-fault factors such as environmental changes and random disturbances, when the value of the energy form feature quantity becomes large, does not necessarily indicate that the fault is degraded, may be caused by non-fault factors, or may be caused by both fault factors and non-fault factors. The traditional method of fault prediction often produces false positives.

3) Traditional trend prediction methods are generally suitable for signal short-term and local time zone ranges. For continuous operation, large-scale and complex equipment with long-running and variable-time operating characteristics, it is often difficult to perform effective faults using traditional and simplified signal analysis methods. forecast.

\section{Predictive maintenance methods for electromechanical equipment based on state monitoring and fault warning information technology}

Equipment maintenance is like a doctor needs to treat after he diagnoses his condition. Equipment maintenance is an important part of ensuring the healthy operation of the equipment and realizing its functions. For high-end, large and critical in-service equipment, the use of traditional after-sales maintenance and regular maintenance methods is not only uneconomical but often avoids accidents. Predictive maintenance is a more scientific and modern maintenance method that comes with the development of computer and information processing technology. The modern predictive maintenance method can implement dynamic condition monitoring according to the actual operating conditions of the equipment and the precursor of the fault and arrange maintenance before the fault occurs. Informatization technology is the key technology to realize predictive maintenance. The information technology of condition monitoring and fault warning of electromechanical equipment can provide technical means and realize ways to realize advanced predictive maintenance methods. In order to improve the level of intelligent automation for predictive maintenance, some research institutes and enterprises have built multi-sensor information acquisition, data mining systems and information fusion systems, and established multi-information databases and information analysis knowledge bases, such as: case bases, map libraries, archives. Libraries (such as historical information bases, including historical maintenance records such as overhaul, downtime, and repair and shutdown), knowledge base (configuration related expert 
systems, knowledge mining tools, knowledge acquisition systems, variable precision rough sets, etc.).

The information unit of the typical predictive maintenance system includes: status information monitoring, multi-source information fusion, useful information mining, fault information acquisition, intelligent information processing, network information sharing, remote information transmission, measurement and control information feedback, equipment information management, maintenance information decision Wait. In order to improve the early warning and predict the efficiency and real-time of the maintenance system, with the development of information network technology, the information service architecture of cloud computing is constructed in the pre-aware maintenance, and the information storage space and information processing speed of massive big data are improved, and the task distribution is calculated. On a large pool of computer resources, users can obtain computing power, storage space and information services; build other related modern information systems (visual systems, Internet of Things systems, and information processing systems such as artificial intelligence, machine learning, pattern recognition, statistics, etc.), for information management.

\section{Research and development of safety monitoring instrument based on state monitoring and fault warning information technology}

For the requirements of multi-class electromechanical system security and early fault information processing, some modern test instrument research units and manufacturing companies usually cooperate with equipment-owned enterprises to carry out research and development of multi-type equipment safety monitoring instrument systems and enterprise predictive maintenance systems. The remote network online monitoring and forecasting center for equipment groups, and continuously improve the information technology level of related instrument systems. In order to adapt to the development of a series of monitoring instrument systems with high technology, multi-variety and small batch characteristics, system integration technology based on information network manufacturing technology is adopted. In order to improve system integration level, integrate R\&D efficiency and expand the scope of application, reconfigurable integration is adopted. The method comprises a modular hierarchical flexible integrated architecture, an intelligent manufacturing and multi-objective optimization mechanism, an information network interconnection and an interface, and corresponding reconfigurable integrated R\&D devices and supporting software packages.

In order to provide advanced equipment for the development of series of monitoring instrument systems, improve the research and development capabilities of monitoring systems, and build an integrated $R \& D$ platform for a series of monitoring instrument systems. The main components of the integrated R\&D platform include: reconfigurable integrated environment and supporting resources (including: multi-sensor signals) System, opto-mechanical device, typical measurement and control laboratory, simulation environment, calibration system, signal processing unit, intelligent analysis unit, information management unit, software and hardware module, interface, network, bus, communication, etc.; Main functions include: Information system integration involves: measurement, acquisition, transformation, processing, integration, transmission, sharing, feedback, results and expression, decision and management, optimization and control. In recent years, typical safety monitoring instrument systems have been widely used in manufacturing, energy, transportation, power, petrochemical, metallurgical, municipal, environmental, aerospace and resource sectors such as: new energy generation, locomotive power, resource extraction, urban water supply, Electromechanical equipment such as coal mine ventilation, gas transmission, high-grade machine tools, aerospace and national defense can realize the safety monitoring and equipment health management of many modern, high-end and key equipment groups in some industries, and also realize the equipment in safe operation area or energy saving Optimized operation under working conditions. 


\section{Electromechanical system state trend prediction technology}

In order to ensure the reliable and effective realization of the functions of electromechanical equipment, the related research work at home and abroad mainly goes through three stages. The first stage: monitoring the equipment operating status; the second stage: conducting fault diagnosis, which is mainly carried out when the fault occurs. Phase 3: Predicting the trend of state development and early failure, and then performing state maintenance, mainly when no failure occurs. State trend prediction technology for electromechanical systems is an in-depth analysis of state monitoring and fault diagnosis techniques. State monitoring and fault diagnosis technology is the basis of state trend prediction technology. Since most of the early faults of electromechanical systems are predictable trend faults, the research on fault trend prediction methods has been carried out at home and abroad in recent years. The following is a numerical analysis method. The method is a more direct prediction method (a method using two-point anomaly states, such as: linear, exponential, etc.); a method using predictive statistics (probability prediction method); Multiplication prediction, regression prediction or causality method (univariate linear regression, multiple linear regression)).2 Modern model prediction method [11]. This kind of method is developing from the research stage to the practical stage (time series prediction model method, gray system prediction model method, etc.).3 Artificial intelligence based prediction method [12]. The method is in the stage of research and discussion (neural network prediction method, expert system prediction method, fuzzy prediction method and chaotic prediction method, etc., and the mutual intersection of these methods).

\section{Conclusion}

With the deepening of the awareness of the protection of electromechanical systems (or electromechanical equipment) and the understanding of equipment maintenance, with the development of monitoring and diagnostic technology and the reduction of application costs, the demand for this technology will also increase. The economic and social benefits brought by technology are becoming more and more obvious, and this technology will be further favored by people. With the development of modern testing technology, computer and network technology, virtual instrument technology, modern signal processing technology and other new technologies, The application of electromechanical system monitoring and diagnosis, electromechanical system condition monitoring and diagnostic technology will be rapidly developed, and will be more widely used in key equipment in many industries such as machinery, petrochemical, aerospace, metallurgy, electric power, coal, nuclear energy, etc. Such as centrifugal pumps, electric motors, engines, generators, compressors, steam turbines, rolling mills, etc. This technology has broad application prospects, and its economic benefits have great potential.

\section{References}

[1] Feng Chenxi, Liu Weidong. Design of Multichannel Signal Simulation System Based on FPGA [J]. Acoustics \& Technology, 2016, 35(4):442-445.

[2] Zhai Chunmei, Fan Ling, Qiu Yang. Comprehensive Research on Intelligent Diagnosis and Forecast Method of Rotating Machinery Faults [J]. Machinery, 2000, 27(1): 21

[3] Jakob Angeby. Estimation signal parameter using the nonlinear instantaneous least squares approach [J]. IEEE Transaction on Signal Processing, 2000, 48(10): 2721-2732.

[4] Brenner M J. Non-stationary dynamics data analysisw ith wavelet-STD filtering [J]. Mechanical Systems and Signal Processing, 2003, 17(4): 765- 786.

[5] He Zhengjia, Cai Gaichang, Shen Zhongjie, et al. Research on equipment operation reliability based on mechanical diagnostic information [J]. China Engineering Science, 2013(1): 9-14. 